\title{
Variationen literarischer Freitodbilder um 1980
}

\section{Variations of Literary Suicide Portrayals around 1980}

\author{
Aleš Urválek
}

\begin{abstract}
This study focuses on selected literary texts, which were created around 1980 and deal with the subject of suicide. An overview-based study attempts to incorporate these texts into the context of the history of German-language literature and to describe the form and function of suicide in these works. Relatively conventional works representing the "Neue Subjektivität" are presented in biographically tuned prose written by female authors (Christa Wolf, Karin Reschke) and Bernward Vesper's novel Reise, which deals with unsuccessfully reconcilitation of the relationship between son and father. The works of Wolfgang Hildesheimer and Botho Strauß are shown as less conventional. The final chapters devote themselves to dramatic (Max Frisch) and prose texts in which suicide is portrayed as part of the events that is concludes in a cycle of eternal repetition or is seen by transhuman optics in which the perspective of individual life is transcended by the perspective of the human race or the development of the planet Earth (evolutionary and geological overlaps).
\end{abstract}

\section{Keywords}

sucide, fascism, women's writing, biographies, autorship, repetition, geology, evolution, anthropocene 
Die deutschsprachige Literatur des 20. Jahrhunderts ist derart reich an Freitod- oder Selbstmordbildern, dass man weniger Probleme damit hat, einschlägige Werke zu finden, als damit, den vorgefundenen Texten eine sinnvolle Struktur zugrunde zu legen. Zunächst aber eine kurze Kostprobe dessen, was das 20. Jahrhundert zum literarischen Suizid insgesamt bietet: Es gibt, wenn man sich auf die deutschsprachige Literatur beschränkt, expressionistische Selbstmörder, die zur Pistole greifen, nachdem ihr Leben gescheitert ist. So geschieht es etwa dem enttäuschten Kassierer in Georg Kaisers Drama Von morgens bis mitternachts (1912, UA 1917), der morgens Geld entwendet und sich abends erschießt, nachdem er eine Desillusion nach der anderen durchgemacht hat. Oder, wenn man schon bei Georg Kaiser ist, begegnet man erlösenden Märtyrersuiziden der Protagonisten, die sich opfern, um ihr Land zu retten, wie man sie aus dem Drama Die Bürger von Calais (1914, UA 1917) kennt, oder aber den sich im kollektiven Selbstmord auslöschenden Massen der Fabrikarbeiter, die trotz etlichen fortschrittskritischen Warnungen von der lebensgefährlichen Gasproduktion nicht ablassen wollen, und trotz der fortschrittskritischen Warnungen immer wieder in die Luft gehen, so zum dritten Mal Georg Kaiser, nun im zweiteiligen Drama Gas $(1918,1920)$. Wenn man etwa in die 1960er Jahre übergeht, stößt man auf einige stellvertretende Freitodaktionen, zu denen es kommt, sobald die Söhne die nazistische Kollaboration ihrer Väter nicht mehr ertragen können, und sich umbringen, um statt der Väter Buße zu tun. Diese Väter können dann entweder reale Naziväter sein, wie es etwa in Martin Walsers Der schwarze Schwan (1964) der Fall ist, oder stellvertretende Väter, so wie Papst Pius der XII für die ganze Kirche in Rolf Hochhuths Drama Der Stellvertreter aus dem Jahre 1963. Zu nennen wären etliche berufliche in Aussicht gestellte Selbstmordaktionen, in der Absicht geplant, die komplette Menschheit vor dem Atomtod zu retten, die aber paradoxerweise nichts bringen, wie man es aus Friedrich Dürrenmatts Die Physiker (1962) kennt. Wechselt man in die Literatur der 1980er Jahre, hat man es mit diversen metaphorischen Freitodarten zu tun, bei denen sich die Romanfiguren beispielsweise in der Textstruktur auflösen, oder die Grenzen unserer Welten ausloten und von diesen Expeditionen nicht mehr zurückkommen. Chrostoph Ransmayrs Romane Die letzte Welt (1988) oder Die Schrecken des Eises und der Finsternis (1984) könnten genannt werden, oder Patrick Süskinds Das Parfum (1985), dessen Protagonist sich am Ende mit sichtlichem Behagen aufessen lässt.

\section{Mit den Büchern in den Freitod}

Für den Einstieg in die Darstellung der literarischen Freitodbilder um 1980 greife ich auf einen bereits um 1935 vollzogenen Selbstmord zurück, auf das berühmte Autodafé, das am Ende von Canettis Die Blendung der weltbekannte Sinologe Peter Kien begeht, der am Ende des Romans beschließt, sich mit all seinen Büchern zu verbrennen. Dieses Beispiel stelle ich voran, da hier vieles vorweggenommen wird, was uns in manchen literarischen Freitodbildern um 1980 begegnen wird. Die allerletzten Romansätze lauten: „Er stellt die Leiter in die Mitte des Zimmers, wo sie früher stand. Er steigt auf die sechste 
Stufe, bewacht das Feuer und wartet. Als ihn die Flammen endlich erreichen, lacht er so laut, wie er in seinem Leben nie gelacht hat" (Canetti 1994: 510).

Für die Kenner Canettis hat dieser Schluss etwas Paradoxes, Irritierendes, gilt dieser Autor doch als ein lebenslanger Todesgegner, jemand, der den Tod sein ganzes Leben lang nicht anerkannt hat; und trotzdem entscheidet sich gerade die Hauptfigur, Hand an sich zu legen. Zudem das laute Lachen der Hauptfigur, das annehmen lässt, dieser Mensch habe wohl erst im Freitod erreicht, was er sich insgeheim gewünscht hatte. Somit macht man schon den ersten Schritt, der bei den literarischen Freitodarten oft zum Tragen kommt: Man wird dafür sensibilisiert, was sich in der inneren Welt des Selbstmörders abgespielt haben mag. Und hier scheint es prekär zu sein, denn Kien ist gegen Ende des Romans nicht mehr fähig, seine innere Welt von der äußeren abzugrenzen, kurz, er wird wahnsinnig. Damit aber der Irritation noch nicht genug: Gerade einer, der sein ganzes Leben lang nur mit Büchern lebt und Bücher liebt, stirbt am Ende gerade auf einem Bücherscheiterhaufen. Er wird, so könnte man es aus seiner Sicht erklären, eins mit dem Einzigen, was er über alles liebt und schätzt. Offensichtlich ist ihm die Welt der Bücher (der Texte) wichtiger als die Welt der Menschen, so dass er, die Grenze zwischen Buch und Mensch missachtend, in den Büchern als Mensch, also nicht nur geistig, sondern auch körperlich, aufgehen will. Was aber nur im (freien) Tode möglich ist, bei dem er mit seinen Büchern eine Art unio mystica erlebt. Erklärungsbedürftig und für unser Thema anschlussfähig ist auch der Umstand, dass ein weltbekannter Wissenschaftler wie der Sinologe Kien trotz seiner Bildung keinen anderen Ausweg weiß, als sich mit seinem ganzen Buchwissen verbrennen zu lassen. Weiter ist zu bedenken, in welchem Ausmaß in seiner Freitodaffinität diverse naturwissenschaftliche Diskurse eine Rolle spielen: Kurz vor dem Selbstmord wird Peter Kien von seinem Bruder aufgesucht, einem modernen Psychiater, der Peter in einem hoffnungslosen Zustand vorfindet, und zur Therapie ansetzt. Diese besteht zunächst mal darin, dass am Beispiel der Ameisen eine Massentheorie geschildert wird, deren Heilprozess für Peter allerdings recht fragwürdig anmutet:

„Die Tiere vergessen - eine ungeheuerliche Erinnerung hat sie gepackt - was sie sind, blinde Zellen eines fantastischen Ganzen. Jeder will für sich sein, bei hundert oder tausend von ihnen fängt es an, der Wahn greift um sich, ihr Massenwahn, die Soldaten verlassen die Eingänge, der Stock brennt vor unglücklicher Liebe [...], der Stock, der vielleicht Ewigkeiten gelebt hätte, die Ewigkeiten, nach denen wir uns sehnen, stirbt, stirbt nach Liebe, an dem Trieb, durch den wir, eine Menschheit, unser Weiterleben fristen! Eine plötzliche Verkehrung des Sinnreichsten ins Sinnloseste. Es ist - man kann das mit nichts vergleichen, ja, es ist, als ob du dich eines hellichten Tages, bei gesunden Augen und voller Vernunft, mitsamt deinen Büchern in Brand setzen würdest. "(Canetti 1994: 474)

Dieser Exkurs wird, wie leicht zu erschließen, seine Wirkung nicht verfehlen. Um das Wesentliche festzuhalten: Als Naturerscheinung befinde sich das Leben stets zwischen Leben und Tod, wobei es als Individuation zwangsläufig im Tod in den Kreislauf der Natur zurückfinde; man suche sich also am Leben zu erhalten, aber genauso stark sei man gedrängt, sich zu entindividualisieren, sich als Individuum im Tode aufzulösen. 
Zieht man nun in recht freier Assoziation zu diesen Feststellungen einige suizidale Texte hinzu, die um 1980 entstanden sind, bietet sich außer dem zentralen Blick auf die Variationen eben dieser Bilder auch die Möglichkeit, sie literaturgeschichtlich zu kontextualisieren, sofern man kritisch überprüft, inwiefern sie den zentralen Stichworten entsprechen, die in den kanonisierten Literaturgeschichten für die späten 1970er und die frühen 1980er reserviert worden sind. Egal, welche der Geschichten der deutschsprachigen Literatur seit $1945^{1}$ man in die Hand nimmt, immer fallen mindestens drei zentrale Begriffe: „Neue Subjektivität“ (in Varietäten wie „Neue Innerlichkeit“, „Neue Sensibilität“), weiter folgt biographische oder autobiographische Literatur, zu der entweder die Nachbearbeitungen der politischen Revolte der 1960er Jahre, oder die sogenannte Väterliteratur zählen, und schließlich die sogenannte Frauenliteratur, ein Schreiben, das sich auf die Suche nach der weiblichen Identität macht.

Ich gehe zunächst auf suizidale Texte ein, die diesen Vorgaben entsprechen, will aber im zweiten Schritt auch auf Texte hinweisen, die im Rahmen der angegebenen literaturgeschichtlichen Rubriken innovativ wirken, oder aber diese ganz unterlaufen. Für typische Frauenliteratur seien im Folgenden diese suizidalen Texte zu halten: Kein Ort. Nirgends von Christa Wolf (1979) und Verfolgte des Glücks (1982) von Karin Reschke. Ein Vaterbuch par excellence insofern, als in ihm die Studentenrevolte und zugleich die Nachwirkungen der nazistischen Vergangenheit verarbeitet werden, stellt Die Reise (1977) von Bernward Vesper dar. Der biographischen Literatur nähern wir uns über Marbot. Eine Biographie (1981) von Wolfgang Hildesheimer an, diverse Varietäten der Innerlichkeit werden anhand der Theorie der Drohung (1975) von Botho Strauß dargelegt, wobei diesen zwei Texten ein großes Innovationspotenzial innewohnt. AbschlieBend wird zumindest ansatzweise auf suizidale Texte hingewiesen, die bisher in den Literaturgeschichten wenig berücksichtigt worden sind, obwohl sie für die Literatur um 1980 nicht weniger ausschlaggebend sind: etwa das das Drama Triptychon (1978) von Max Frisch, oder die prosaischen Texte Strahlender Untergang (1982) von Ch. Ransmayr, Der Mensch erscheint im Holozän (1979) von Max Frisch oder Rumor (1980) von Botho Strauß.

\section{Wegen des Vaters in den Freitod}

Der autobiographische Romanessay Die Reise, verfasst von Bernward Vesper, dem Sohn von Will Vesper, einem völkischen NS-Dichter, wurde 1977, also postum ediert, nachdem Bernward, der einige Jahre mit der RAF Terroristin Gudrun Ensslin gelebt hatte, Selbstmord begangen hatte. Der Romanessay besteht aus mehr als 600 Seiten langen Tagebuchnotizen, die ein völliges Scheitern der radikalen Linken schildern, die vergeblich auf die Unterstützung der Massen gehofft haben. Einen großen Raum nimmt darin der verhasste Vater Bernwards ein, an dessen Schatten sich der Sohn abarbeitet, dabei im-

1 Vor allem: SCHNELL, Ralf (1993): Geschichte der deutschsprachigen Literatur seit 1945. Stuttgart: Metzler; HOFFMANN, Dieter (2006): Arbeitsbuch. Deutschprachige Prosa seit 1945. Tübingen Basel: A. Francke. Differenzierter werden die 1970er Jahre behandelt in: BARNER, Wilfried, Hg. (1994): Geschichte der deutschen Literatur von 1945 bis zur Gegenwart. München. C.H. Beck. 
mer verbissener und mehr und mehr irrewerdend, sofern er nie frei zu werden vermag, sondern immer nur ex negativo wiederholt, was der Vater gemacht hatte. Das komplette Scheitern seines Lebensentwurfes führt Bernward 1971 in eine psychiatrische Klinik, wo er sich mit einer Überdosis Schlaftabletten das Leben nimmt. Somit ist Vespers Roman allem voran ein Buch der Rebellion „gegen den Krieg, die Manipulation, die Verführung, die Vergeudung der Jugend, der Begeisterung, des Elans, der Hoffnung“ (Vesper 1983: 55); ein Buch des Hasses auf die Väter, die ihre Söhne ums Leben, also um „Träume, um Liebe, Geist, Heiterkeit, ums Ficken, um Hasch“ (ebenda) gebracht haben. Wohl auch darum wird hier der Selbstmord immer wieder als eine durchaus naheliegende Potentialität häufig imaginiert (vgl. etwa Vesper 1983: 20). In einer dieser postmortalen Imaginationen stellt Vesper seinen Körper dem Anatomischen Institut der Goethe Universität in Frankfurt zu Verfügung, um dessen Reste danach „stillschweigend dem Kreislauf der Materie, der sie entstammen, wieder eingliedern“ (Vesper 1983: 529) zu lassen.

Dem herbeibeschworenen und dann tatsächlich realisierten Selbstmord scheint keine besondere, ja unnatürliche Komponente anzuhaften, in ihm kulminiert vielmehr ein Leben, das an seinen eigenen Voraussetzungen und Dispositionen gescheitert ist, so sehr sich der Protagonist dem entgegenzustellen gesucht hatte. Anders als im Text kommt indes dem Selbstmord auf der Ebene, auf der es um die Unmittelbarkeit der Aussage geht eine besondere Qualität zu: Gerade er soll nachträglich die Authentizität dieses Romanessays garantieren, dessen Autor, so der Begleittext, Literatur als Harakiri betreibe. Da es sich hier um Aufzeichnungen eines komplett gescheiterten Protagonisten, in dessen persönlichem Scheitern immer auch das kollektive Scheitern der ganzen betroffenen Generation gespiegelt schien, handelt, mag eben der Selbstmord hier ausschlaggebend dafür gewesen sein, dass dieses Buch in die Literaturgeschichten als ein mit Blut geschriebenes Kultdokument seiner Zeit eingegangen ist.

\section{Mit Kleist in den Freitod}

Anders in den Texten von Christa Wolf und Karin Reschke; in beiden wird - durchaus im Sinne der weiblich akzentuierten Innerlichkeit - das Schicksal emanzipierter und äußerst empfindsamer Frauen aus dem Umkreis der Romantik geschildert, mit einem Freitod am Ende. In Kein Ort. Nirgends begeht den Selbstmord Karoline von Günderrode, eine recht bekannte romantische Dichterin, die von ihrem verheirateten Liebhaber (Friedrich Creuzer) das Versprechen erhält, er würde seine Frau eines Tages verlassen. Doch als diese schwer erkrankt, wird aus dem einstigen Versprechen ein anderes, eben die Geliebte, Karoline von Günderrode, zu verlassen. Was folgt, ist unschwer zu erraten: Günderrode versetzt sich einige tödliche Dolchstiche unter die Brust.

Karin Reschke greift in Verfolgte des Glücks einen wohl noch bekannteren Suzidfall der deutschen Literaturgeschichte auf: Henriette Vogel, eben die Frau, die sich, damals schon unheilbar krank, bereit erklärt hat, mit Heinrich von Kleist einen gemeinsamen Doppelsuizid zu begehen. Zwei historische Frauenfiguren werden nun für zwei weibliche Autorinnen zur Projektionsfläche für ihre Suche nach weiblicher Selbstbestimmung, 
geradezu zur „Selbsterkundung im historischen Kostüm“ (Magenau 2002: 215). Zudem verfügen beide Texte mit Heinrich von Kleist über eine gemeinsame männliche Projektionsfigur, der für die Protagonistinnen jeweils einen Suizid-Verbündeten darstellt. Während im Falle von Henriette Vogel diese suizidale Begegnung historisch verbürgt und der Doppelselbstmord belegt ist, ist die Begegnung zwischen Heinrich von Kleist und Karoline von Günderrode von Christa Wolf erfunden worden, die zwei dürften sich nur flüchtig gesehen haben.

Die von Christa Wolf geschilderte Wahlverwandtschaft zwischen Kleist und Günderrode steht von Anfang an deutlich im Zeichen des Selbstmordes, der zunehmend mit Andeutungen herbeiimaginiert wird. Kleist und Günderrode werden somit nicht nur einander, sondern auch dem Suizid nähergebracht: „Sie kennt die Stelle unter der Brust, wo sie den Dolch ansetzen muss, ein Chirurg, den sie scherzhaft fragte, hat sie ihr mit einem Druck seines Fingers bezeichnet“ (Wolf, 1981: 9) geht ihr durch den Kopf in Anwesenheit von Kleist. Und wenig später geht sie auf ihn zu und plötzlich rutscht etwas Glitzerndes heraus und "gleitet über das glatte Parkett. Sehr seltsam: ein Dolch. Kleist, geistesgegenwärtig, hebt die Waffe auf, reicht sie der Günderrode“ (Wolf 1981: 45).

Überdeutlich wird der Text, der existentiellen Krise der Autorin entsprungen, zu einer doch alternativen Möglichkeit für diejenigen, die ähnlich wie Christa Wolf „mit dem Rücken zur Wand stehend“ (Magenau 2002: 291) mit dem Selbstmodergendanken den Anspruch an Freiheit und Wahrheit verbinden. Der Freitod wird hier als eine nun auch den Frauen zustehende Möglichkeit in den Raum gestellt; das Recht einer Frau, Hand an sich zu legen wird mit dem Recht auf Individualität, auf künstlerische Entfaltung analogisiert. Kleist erscheint hierbei als der geistige Wegweiser für alle sensiblen, mutigen, emanzipierten und wahrheitssuchenden Frauen, die ihm in all diesen Qualitäten ebenbürtig sind.

Karin Reschkes Verfolgte des Glücks hält sich freilich an den belegten Fall des Doppelselbstmordes Kleist-Vogel, wobei der Text bis auf den notorisch bekannten Abschiedsbrief „Mein Heinrich, mein Süßtönender“, auf den es hier hinausläuft, auf fiktiven Tagebuchnotizen Vogels basiert. Zu tun hat man es also mit dem Tagebuch einer Verfasserin, die, was man bereits vor der Lektüre weiß, am Ende des Textes Selbstmord begehen wird. Diese Notizen sind von Reschke nicht vorgefunden, sondern erfunden worden. Sie beginnen am 3. 4. 1798 (sterben wird Henriette am 21. 11. 1811), der ersten Eintragung wird noch eine Passage vorangestellt, in der Kleist das Tagebuch von Henriette in die Hand gedrückt bekommt: Daraus resultiert der Effekt, dass man die Tagebuchnotizen sozusagen gemeinsam mit dem Lektor Kleist liest, der die Notizen wie auch die Verfasserin nicht überleben wird.

Nicht anders als bei Wolf ist der Tod von Anfang an überpräsent; den Kindern werden grausame Geschichten erzählt, in denen etliche Köpfe abgeschnitten werden, Henriettes Vater wird von seiner ehemaligen Gattin mehrmals zu Tode verflucht und vieles mehr. Die zentrale weibliche Figur wird in einer ähnlich alternativlosen Lage erfasst: unglücklich verheiratet, mit Händen im Schoss, unschlüssig etwas Sinnvolles zu tun, in der Ehe einfach nur dahinlebend. Mit der Hochzeit ist sie in das Leben ihres Mannes eingezogen, ohne ihr eigenes zu gewinnen oder zu haben. Derart ratlos bekommt sie 
eines Tages von ihrer Freundin ein Buch geschenkt, das man doch irgendwie erwartet: Werther. Die Lektüre macht ihr ihre hoffnungslose Lage deutlich:

Wie soll ich ihm andeuten, dass ich mich innerlich von ihm entfernt hatte? Wie ihm andeuten, dass die unzähligen Überfälle des Nachts, die rohe Gewalt hinter den verschlossenen Türen, ohne Licht, ohne Worte, ohne Zartgefühl, meine Empfindungen aufs Äusserste verletzt haben? Wie ihm begreiflich machen, dass ich keine Zuneigung mehr für ihn, keine Begierde verspüre, seit er mir in einem düsteren Augenblicke den Hals mit seinem ganzen Gewichte zugedrückt und mich mit jäher Macht genommen? (Reschke 1982: 124)

Nun läuft es nur noch auf eins hinaus: Es dauert nicht lange, bis ihre psychischen Probleme zu somatischen werden. In ihrer Gebärmutter werden die aufgestauten Emotionen zu einem Klumpen, der krebsartig anschwillt und alles andere verfaulen lässt. Von der Wertherlektüre sensibilisiert, mit dem schweigsamen Herrn Kleist schnell verbündet, und an die Entscheidung herangeführt, dem Leben gemeinsam ein Ende zu setzen, gehört auch diese Protagonistin zu denjenigen, die mit der äußersten Entscheidung zum Freitod zugleich Freiheit in kaum gekanntem Ausmaß für sich in Anspruch nehmen. Dass diese Entscheidung eine bereits unheilbar kranke Frau trifft, scheint daran nicht viel zu ändern. Sie verlässt mit Kleist ihr vertrautes Heim samt ihrem Ehemann, doch nicht, um mit einem anderen Mann zu leben, sondern, um mit ihm zu sterben. Mit der Entscheidung zum Freitod befreit sie sich ein für alle Mal. Somit fügen sich beide präsentierten Suizidtexte gut in das Bild der weiblichen Literatur der 1970er Jahre; der hier vollzogene Schritt zum Freitod wird als bewusst bejahter Schritt zur Freiheit, als Ausweg aus einer bereits alternativlosen Situation dargelegt, durch den man zur letztmöglichen authentischen Selbstbestimmung findet. In ihm wird die emanzipatorische Parole dieser Literatur der Mensch meines Lebens bin ich bis zur äußersten Grenze, bis in den Tod hinein vollzogen.

\section{Freitod der Biographie}

In Wolfgang Hildesheimers Marbot. Eine Biographie hat man es erstens mit einem männlichen Suizid zu tun, der, zweitens, die Rubrik biographische Texte recht innovativ überschreitet, wenn nicht sprengt. Denn Hildesheimer liefert mit dieser suizidalen Biographie einen Text, der die für Biographien konstitutive referentielle Funktion in Frage stellt. Zugespitzt gesagt, diesen Text könnte man für eine durchaus ironische Antwort eben auf die große Welle solcher Biographien der 1970er Jahre halten, die eben in den gerade erwähnten neusubjektivistischen biographischen Texten über Günderrode, Vogel vorgestellt wurden.

Um das Innovative zu erfassen, sei Hildesheimers Text in zwei Strängen abgehandelt: Im ersten wird es um Genrefragen, im zweiten um Suizid gehen. Marbot steht deutlich am Ende eines Reflexionsprozesses, dessen Verlauf zwei Befunde charakterisieren: Die wahre Biographie müsse ihren Helden erfinden, der Biograph sei ganz Erzähler, 
der Erzähler sei ganz Biograph (Neumann 1986: 30). Und eine Biographie könne man nur über eine Figur schreiben, die niemals existiert habe (Hildesheimer 1981b). Auf den Kopf gestellt wird hier, was man über Biographie gewöhnlich denkt: Biographie erfinde ihren Helden nicht, der Biograph sei eher ein Dokumentarist, er setze an Referentiellem an und künstele daraus eine Biographie hervor. Nicht so bei Hildesheimer: Der Prozess, an dessen Ende mit Marbot. Eine Biographie eine Biographie steht, bei der schon der Untertitel (Biographie) deutlich fiktionaler Prägung ist, und die zentrale Figur (Marbot) nur innerhalb dieser Biographie existiert, ist folgender. In den 1970er Jahren schreibt Hildesheimer an einer Biographie über Mozart, noch mit dem Ansatz, nichts erfinden zu wollen. Die Hypothese lautet: Mozart würde man biographisch aus den Augen verlieren, wenn man ihn mit Eigenem ausstaffieren würde. Darum gelte es, den Abstand zu der porträtierten Figur beizubehalten, jede Mutmaßung des Biographen kenntlich zu machen, Widersprüche nicht wegzuglätten. Das heißt: Hildesheimer, der hier scharfsinnig das Verhältnis von Biographen und Biographiertem auslotet, indem er die oft überschrittenen „Grenzen von spekulativem und wissenschaftlichem Verfahren deutlich“ (Klein 2009: 344) macht, gesteht ein, dass er als Biograph seine Mutmaßungen über den Biographierten möglichst ausblenden muss. Da man allerdings als Biograph um die eigene Subjektivität nicht herumkommt, bleibt ein einziger Ausweg übrig: die eigene Subjektivität nicht zu verstecken, sie vielmehr einzugestehen und zu betonen. Die Mozart-Biographie Hildesheimers ist also betont widersprüchlich, darum wurde sie vom Verfasser als eine negative Biographie, als ein Werk des biographischen Scheiterns bezeichnet: „[...] wenn es mir gelungen ist, etwas von dieser Unvorstellbarkeit zu vermitteln, zu demonstrieren, dass Mozart nicht einer von uns ist, dann ist mein Buch gelungen." (Hildesheimer 1988: 138)

Aus der Einsicht in die Unmöglichkeit einer nichtfiktionalen Biographie resultiert nicht nur der Freitod der konventionellen Biographie, sondern auch das Gebot, sich im fiktional Biographischen zu versuchen. Und eine literarisch-fiktionale Biographie, die allerdings seinen biographischen kunsthistorisch-faktualen (vgl. Klein 2009: 84) Status herausstellt, ist eben Marbot. Der Hauptprotagonist Andrew Marbot ist ein Kunstkenner, Kunstinterpret, aber kein Künstler. Um ihn ist ein Netz von fingierten Fakten und Dokumenten aufgebaut, die aber meist an historisch beglaubigte Persönlichkeiten und Ereignisse gebunden sind. So begegnet Marbot intellektuellen Größen wie Goethe, Schopenhauer oder William Blake, es werden Berichte darüber zitiert wie auch anderes Material, das die referentielle Funktion des Textes beglaubigen soll. Darunter auch eine freilich erfundene wissenschaftliche Biographie über Marbot aus dem Jahre 1888, sowie das Bildmaterial mit Portraits, die den Text begleiten, und, wie sich für eine Biographie ziemt, auch ein Personenregister, das das sämtliche Personal mit Lebensdaten umfasst, freilich mit einer Lücke bei dem Buchstaben M: Der Name Andrew Marbot sowie der seiner Eltern Catherine Marbot und Francis Marbot, von denen im Buch sogar Porträts abgebildet sind (angeblich von Henry Raeburn in den Jahren 1803 und 1804 gemalt) fehlen, denn die hat es samt den Bildern jenseits dieses Textes nie gegeben. Nur am Rande bemerkt: So ganz neu ist dieses Verfahren bei Hildesheimer nicht. Ausprobiert wurde es bereits 1952 in einer Erzählung des Bandes Lieblose Legenden, wo Hildesheimer 
sämtliche Werke, Quellen, Briefe eines gewissen Gottlieb Theodor Pilz komplett erfunden hat, eines Mannes, der nicht wie Marbot Kunstwerke deutet, sondern sich darum bemüht, Kunstproduktion zu bremsen: Dieser erfundene Pilz redet eben mit großem Erfolg den Autoren ihre geplanten Werke aus, die dann nie erscheinen. Auf seine kunsteindämmende Aktivität sei zurückzuführen, dass etwa F.J. Jahn seinen Dramenzyklus über die Germanenkämpfe nie publiziert habe, Beethoven zwischen 1814 und 1818 eine unproduktive Phase hatte, Mendelssohn und Schumann bei vier Sinfonien geblieben sind und vieles mehr. Ein genialer Witz, der auch weiter aufgegriffen wurde, stößt man doch im Killy LiteraturLexikon auf einen diesem Pilz gewidmeten Artikel.

Aber zurück zu Marbot: Die Brücke zwischen dem Suizid und den technischen Fragen der Biographie stellt Marbots Mutter dar, mit der Andrew, so die Biographie, lange Jahre in einem zufriedenen, obwohl inzestuösen Verhältnis gelebt hat. Die an sich schon erfundenen Quellen, aus denen nun zitiert wird, seien, so heißt es, bevor sie zum Text geworden sind, nochmal durch die Hände Marbots Mutter gegangen. Das macht das Spiel mit dem Genre der Biographie noch spannender und komplexer, insofern nahegelegt wird, eine der an sich erfundenen Figuren hätte etwa aufgrund der Eifersucht einige Dokumente (darunter Briefe, die Marbot seinen anderen Geliebten geschickt hat) gefälscht oder sogar zerstört, so dass dann die (an sich nur vorgetäuschte) Dokumententreue nochmals gebrochen wird. Alles in allem war Marbots Leben, obwohl recht interessant und bunt, prekär und nicht zufriedenstellend: zwar genial, aber nur als Kunstinterpret, der sich in andere kongenial einzufühlen wusste, war er kein schaffender Künstler. So stellt der Suizid am Ende seines Lebens, im Gegensatz etwa zu dem der Günderrode oder Vogel, einfach keinen Schritt über das Gegebene hinaus dar, der eine Wandlung des sich zum Freitod Entschließenden implizieren würde, vielmehr mutet er wie eine natürliche Fortsetzung des Lebens an, das vom Kreislauf des ewig Halbschöpferischen ermüdet worden ist. Darum auch die Absenz des Dramatischen und Heroischen, die sich etwa in einem Abschiedsbrief äußern würden. Marbot verlässt schlicht eines Tages das Haus, ohne von jemandem erblickt zu werden, ja ohne jegliches Dokument hinterlassen zu haben, das signalisieren würde, hier wurde dem Leben freiwillig ein Ende gesetzt. Der Freitod geschieht gleichsam beiläufig, selbst die den letzten Schritten innewohnende inszenierte Außergewöhnlichkeit des Abschiednehmens fehlt. Es wird weder etwas demonstriert noch eine Botschaft vermittelt. Still und diskret, ohne jegliche Körperspuren zu hinterlassen, verschwindet Marbot aus der Textwelt, nur die fehlende Pistole aus seiner Schublade lässt darauf schließen, was sich hier ereignet hat. Marbots Leichnam wird nie gefunden.

Dazu zwei kurze Bemerkungen: Dieses Verschwinden aus dem Leben/Text nimmt literaturgeschichtlich gesehen viele (Reise)Romane der 1980er Jahre vorweg, deren Hauptfiguren etwa bei ihren Polarexpeditionen verschwinden. Neben Sten Nadolny wird sich insbesondere Christoph Ransmayr als ein Experte des spurlosen Verschwindens ausweisen, wovon seine Romane Die Schrecken des Eises und der Finsternis (1984) oder, in anderen Zeiträumen angesiedelt, Die letzte Welt (1988) zeugen. Und: Hildesheimers Reflexionen der Biographie lassen sich plausibel mit dem Stand der Autobiographiereflexionen verknüpfen, die an ungefähr zeitgleichen Texten expliziert wurden. Seitdem 
etwa Texte wie Max Frischs Montauk (1975) oder die dreiteilige Autobiographie von Elias Canetti (Die gerettete Zunge [1977], Die Fackel im Ohr [1980], Das Augenspiel [1985]) erschienen sind, kann man die Autobiographie kaum mehr für eine Dokumentation des an sich vorliegenden Lebens halten. Sie sei eher als erinnernde Neuschöpfung zu bezeichnen, bei der der fiktionale Anteil des Verfassers unumstritten ist, darum nicht ausgeklammert, sondern mitthematisiert werden sollte. Somit partizipiert Hildesheimers Text recht innovativ daran, was seitdem für moderne Bio- und Autobiographien konstitutiv, und was, in diversen Ausprägungen vielen doku- und metadokumentarischen Romanen der sogenannten Postmoderne eigen wird.

\section{Doppelter Freitod}

Im selben Jahr wie Montauk von Max Frisch ist auch Theorie der Drohung von Botho Strauß erschienen, ein früher und eher weniger bekannter prosaischer Text des berühmten und umstrittenen Dramatikers, Prosaikers und Essayisten. Seine frühen prosaischen Texte (Marlenes Schwester [1975], Theorie der Drohung [1975], Die Widmung [1977], Rumor [1980]) sind voll von besonderen Individuen. Diesen Autor interessiert gerade die Schwelle zwischen dem, was für die Figuren das Eigene, und was für sie das Fremde ist; er ist ein Spezialist für den Raum, in dem die Figuren zwischen das Bewusste und Unbewusste, Bejahte und Verdrängte, Erwünschte und Befürchtete gespannt sind. Darum sind die Figuren spezifisch: Es sind keine klassischen runden Figuren mit greifbaren Grenzen, vielmehr sind es „Nicht-Personen eines äußerst porösen Charakters, Inneres und Äußeres werden eins“ (Demetz 1988: 278). Sie sind nach außen geöffnet, durchlöchert; sie sind weniger selbständig agierende Subjekte, vielmehr Durchgangstationen für Regungen, Gefühle, Texte, Worte, Codes. Bereits der erste Text von Strauß, Marlenes Schwester, zeichnet das Bild einer Frau nach, die nicht fähig ist, gesellschaftlich sowie privat zu leben, weil sie stets in absolute Abhängigkeit zurückfällt. Immer wieder wird sie mit ihrer Wunschangst konfrontiert, sich in der Summe der sie bevölkernden Stimmen aufzulösen; das heißt der Freitod wird von ihr immer wieder erwogen, und ambivalent empfunden, einerseits als Versagen, andererseits als Erlösung aus den Lebensqualen. Aber sie vollzieht den Schritt nicht, bleibt unentschlossen. Nichtsdestotrotz wende ich mich im Folgenden der Theorie der Drohung zu, wo auf den ersten Blick kein Freitod zu sehen ist.

Der Text beginnt am ersten Kalendertag des Winters mit einem schweren Schneefall, der die tägliche Landschaft des Erzählers spurlos verschwinden lässt. Alles bisher Bekannte und Orientierungstiftende wird von einer weißen Schneedecke bedeckt; alles scheint ihm abhandengekommen zu sein. Der müde gewordene Autor stellt sich nun zur Aufgabe, das „eigene Ungeschriebene und die unüberblickbare Gesichtslosigkeit der Natur“ (Strauß 1975: 43) unzertrennlich ineinander abzubilden. Daraufhin schließt er sich von der Welt ab und beginnt seinen eigenen Stimmen zu lauschen. Gleich in der ersten Szene seines zur Geschichte gewordenen Selbstgesprächs ist der Ich-Erzähler in einem innerlich gespaltenen Zustand. Obwohl nicht die geringste Hoffnung besteht, 
dass er angerufen werden wird, richtet er alle seine Aufmerksamkeit in die Erwartung eines Telefonanrufs. Er beobachtet den Telefonapparat, hebt den Hörer ab, um sich zu überzeugen, dass das Telefon in Ordnung ist, und plötzlich hört er dort eine fremde Stimme, die ihn gerade begrüßt. Die erzählende Stimme scheint mit der ihr fremden Stimme, von der sie angerufen wird, in unbewusster Verbindung zu stehen.

Angerufen wird der Ich-Erzähler von einem Psychiater, der in der Klinik eine Kranke habe, die dauernd den Namen des eben Angerufenen schreie. Dieser fährt ins Krankenhaus, wo er eine junge Frau vorfindet, die nach wie vor seinen Namen ruft. Er stellt gleich fest: Diese Frau sieht er zum ersten Mal in seinem Leben. Doch obwohl ihm diese Frau völlig unbekannt ist, erscheinen ihm ihre Rufe langersehnt; er fühlt sich durch sie an seine „Notizen zur Wunschangst“ erinnert, in denen eine ähnliche weibliche Figur „plötzlich aufgetaucht war und dort das flüchtige Fragment einer wirklich hoffnungsvollen Begegnung hinterlassen hatte" (Strauß 1975: 46). Für die ganze Erzählung bleibt der „Zwischen-Raum“ ausschlaggebend, in dem der Ich-Erzähler nicht weiß, obwohl er irgendwie ahnt, in dem er wünscht und zugleich befürchtet, in dem er angesprochen (angerufen) wird und zugleich selbst spricht, in dem er schreibt, indem es in ihm schreibt. Das Wesentliche des Textes spielt sich an der Schwelle zwischen der eigenen Stimme, der fremdgewordenen Stimme und Stimmen, die in einem literarischen Text sprechen.

Was korrespondiert dem auf der Handlungsebene? Der Ich-Erzähler soll sich mit einer Frau treffen, die er zwar nicht kennt, nach deren Stimme er sich jedoch seit langem sehnt. Diese Frau beharrt darauf, mit ihm in den Jahren 1968-1970 zusammengelebt zu haben, woran er sich nicht erinnern kann, da er in diesen Jahren mit einer gewissen S. gelebt haben will. Somit wird der Erzähler mit zwei Frauen konfrontiert: Die eine, Lea, behauptet, mit ihm gelebt zu haben, ja Zugang zu seinen Erinnerungen zu besitzen. Die andere, die S. heißt, was man wohl auch als Es verstehen könnte, ist eine, mit der er gelebt hat, an die er sich aber kaum erinnern kann. Nahe liegt die Annahme, jede der Frauen stünde für einen anderen Lebensmodus: Lea scheint von der Vergangenheit des Erzählers auch das zu wissen, was er selbst nicht wissen will oder kann. Mit Lea scheint ihm dieser Mehrwert, das eigene Ungeschriebene, Verschwiegene, unbewusste Gebliebene und Ungewisse entgegenzutreten; all das, was es mit S. geben konnte, ohne dass „Es“ ihm zu Bewusstsein gekommen wäre. S. würde somit für Jahre stehen, die man wie im Es-Modus, also irgendwie unbewusst verbracht hat, Jahre, in denen man nur schwerfällig tagelang gelesen, unendlich diskutiert, theoretisiert und psychologisiert hat; die Schattenseite der Jahre der Studentenrevolte.

Mit Lea versucht nun der Erzähler weiter zu leben, was für ihn, da er Schriftsteller ist, heißt, seinem Beruf nachzugehen, an seinem Buch zu arbeiten, das wenig überraschend auch „Theorie der Drohung“ heißt. Doch diesen Schreibprozess begleiten seltsame Irritationsmomente. In den vorher verfassten Notizen zu dieser Theorie kann sich der Ich-Erzähler kaum wiedererkennen. So stellt er nach und nach fest, seine Notizen, seine Texte, seine Stimme seien ihm fremdgegangen, dürften also nicht (nur) von ihm verfasst worden sein. „Wer, um Himmels willen hat mir das in mein Buch geschrieben“ (Strauß 1975: 70), ruft er erstaunt aus. Es mutet beinahe so an, als hätte er als Romanfigur eine poststrukturalistische Wende durchgemacht, bei der ihm deutlich wird, dass von seinem 
Subjekt das ganz Andere nicht wegzudenken ist: „Was ich auch schreibe, es schreibt über mich. Ich schreibe unaufhörlich den Fremden, der mich bedroht“ (Strauß 1975: 72), heißt es weiter unten. Als Autor wird er Zeuge seiner eigenen poststrukturalistischen Destruktion sowie auch der Text an sich als Orbis Pictus der zentralen poststrukturalistischen Theoreme lesbar wird: Aus dem selbstmächtig geglaubten Text wird ein Plagiat, aus dem Autor ein Abschreiber, ein Kopist, eine Null-Person, diese Durchgangsstation aller möglichen Literatur. Doch damit noch nicht genug: Nachdem seine Version der „Theorie der Drohung“ fertig ist, geht er in die Bibliothek, wo sein Blick zufällig auf ein anderes Buch fällt, das gerade ein anderer zurückgegeben hat: Der Titel dieses Buches lautet „Theorie der Drohung“; selbst der Titel seines Buches ist also ein Plagiat.

Als Autor mehrmals vom Sockel gestürzt, der souveränen Autorschaft beraubt, erblickt der Ich-Erzähler von nun an die einzig mögliche Rettung in der absoluten Theorieabstinenz. Er verzichtet auf jede Reflexion über die Theorie und die Gegenstände seines Lebens, um sich gnadenlos Lea preiszugeben. Er kann aber nicht umhin, sein Leben mit Lea zugleich in der Schrift zu verdoppeln; eine Liebesgeschichte, die nicht unbeschrieben bleiben darf. Dies wagt er, die poststrukturalistische Lektion hat er schon hinter sich, sogar auf die Gefahr hin, von Lea, ja von seinem Text über seine Liebe zu Lea fremdgeführt, also verachtet, verspottet, überschrieben oder vereinnahmt zu werden. Definitiv jetzt ist sein Freitod besiegelt: Sobald das Buch über Lea fertig ist, löst sich, da vom Text verzehrt, Lea als Figur der Geschichte auf. Dieser Dialektik, um konsequent zu sein, darf auch er nicht entgehen. In der Tat: Aus ihm wird, was er insgeheim immer gewünscht, wovor er aber auch Angst hatte. Das Buch ist zwar fertig, die Autorschaft allerdings wird der Erzähler abgeben müssen. Als am Ende des Buches Lea nochmals auftaucht, tut sie es nur, um dem Erzähler die Autorschaft zu entreißen. Sein Wunsch, das Buch über seine Liebe zu Lea mit dem Titel „Theorie der Drohung“, geht in Erfüllung, doch nur um den Preis, dass er nicht dessen Autor sein darf. Sein mutiger Versuch, das Andere seiner selbst zu schreiben, endet damit, dass er zwangsläufig einen doppelten Suizid begeht: einmal als Autor, der hinter seinem Text verschwindet und die Autorschaft an die Andere abgibt. Und einmal als Figur, die, so die letzte Szene, verschwindet, besser die Gestalt von Lea annimmt. Als er sich in der letzten Romanszene im Spiegel sieht, stellt er fest: „Ja es war ihre Frisur, ihre Art, die Haare über den Schläfen hochzustecken, es war auch ihr Make-up, die dunkelgrünen Lidschatten, die clownhaft breit geschminkten Lippen; und ich trug ihr zerschlissenes Chiffonkleid unter dem Mantel. Was ich mir immer gewünscht hatte, brauchte ich nicht länger zu suchen. Ich war Lea." (Strauß 1975: 105)

Man liest also eine Geschichte, in der sich das erzählende Ich von einer der Figuren seiner Geschichte umbringen lässt, was insofern als ein Freitod bezeichnet werden kann, als dieser Tod aus der Logik des Schreibprozesses heraus ein erwünschter ist. Und sein Wunsch, die geliebte Lea nach wie vor betrachten zu können, geht ähnlich gebrochen in Erfüllung. Er wird sie zwar lebenslang (obzwar nur im Spiegel) sehen können, ohne allerdings er selbst bleiben zu dürfen. Ein doppelter Freitod, der in der Verwandlung aufgehoben wird. 


\title{
6. Wenn selbst der Freitod keine Lösung ist
}

Aus der Fülle der einschlägigen Dramentexte um das Jahr 1980 sei ein eher weniger bekannter ausgewählt. Max Frischs Spätdrama Triptychon. Drei Szenen (1979) ist ein düsterer Text, in dem man das Schattenreich betritt, das allerdings anders ausschaut, als man es sich gewöhnlich vorstellt. Einfach gesagt: Frisch entwirft ein Jenseitsbild, das am ehesten an die Vision der „Ewigen Wiederkunft des Gleichen“ erinnert, so wie sie F. Nietzsche in Die fröhliche Wissenschaft formuliert hat:

\begin{abstract}
Wie, wenn dir eines Tages oder Nachts, ein Dämon in deine einsamste Einsamkeit nachschliche und dir sagte: „Dieses Leben, wie du es jetzt lebst und gelebt hast, wirst du noch einmal und noch unzählige Male leben müssen; und es wird nichts Neues daran sein, sondern jeder Schmerz und jede Lust und jeder Gedanke und Seufzer und alles unsäglich Kleine und Grosse deines Lebens muss dir wiederkommen, und Alles in der selben Reihe und Folge - und ebenso diese Spinne und dieses Mondlicht zwischen den Bäumen, und ebenso dieser Augenblick und ich selber. Die ewige Sanduhr des Daseins wird immer wieder umgedreht - und du mit ihr, Stäubchen vom Staube! (Nietzsche 1954: 202)
\end{abstract}

Das Düstere an dem Text von Frisch ist, dass diese niederschmetternde Vorstellung vom ewig Gleichen, die schon fürs Diesseits schlimm genug ist, auch dem Jenseits zugrundegelegt wird; da wo man üblicherweise Entlastung, oder zumindest etwas Anderes erwartet, waltet das immer Gleiche, herrscht die Ewigkeit des Gewesenen. In diesem Schattenreich geschieht einfach nichts, was nicht schon geschehen ist. Nicht nur die lebenden, sondern auch - und vor allem - die toten Protagonisten sind dazu verurteilt, ihr Leben mit ihren deprimierenden unendlichen Wiederholungen desselben und desgleichen unendlich durchzumachen. Was für unser Thema nicht ohne Brisanz ist: In diesem Drama verliert selbst der Freitod letzte Anzeichen irgendwelcher subversiven Kraft. Täuscht sich, wer von ihm allein minimale Änderung erwartet. Denn auch er sowie alles, was vor und nach ihm liegt, unterliegt demselben Kreis der Wiederholungen, dem nicht zu entkommen ist. Ihm unterliegt auch die Struktur des Dramas, das letztendlich alle Erwartung der Figuren, es gäbe irgendwann, sei es nach dem Tode, sei es in der nächsten Szene, einiges, was Änderung bewirken könnte, auslöscht. So gibt das Drama mit seiner zukunftslosen Struktur ein Bild des hoffnungs-, und zukunftslosen Lebens ab, das sich selbst nach dem Tod auf die Zukunft fassen muss, die es schon zur Genüge kennt. Darum stellt auch der verzweifelte Freitod einer der Figuren in der letzten Szene kein Ende des Dramas dar, sondern vielmehr den Anfang, von dem an es von Neuem losgehen muss. Dieser Freitod scheint gegen die Ewigkeit des Gewesenen machtlos zu sein.

\section{Mit dem Freitod über den Menschen hinaus}

Zum Schluß noch ein kurzer Blick auf drei Texte, denen bisher in den Literaturgeschichten eher weniger Aufmerksamkeit gewidmet wurde. Christoph Ransmayrs Erstlingswerk Strahlender Untergang (1982) steht deutlich im Schatten Ransmayrs bekannterer 
Romane. Max Frischs Der Mensch erscheint im Holozän (1979) ist wohl der Text, zu dem sich die Frisch-Forschung verhältnismäßig am wenigsten geäußert hat. Und schließlich Rumor (1980) von B. Strauß, dem in meinen Augen kaum die Aufmerksamkeit gewidmet worden ist, die dieser Text verdient. Zudem bieten sie uns die Gelegenheit, den letzten der Freitodaspekte zu erwähnen, die hier in freier Assoziation zum Text Cannettis verfolgt werden. Nach Texten, in denen Freitodbilder oft nicht nur als irritierende, vielmehr als insgeheim gewünschte dargestellt wurden, die größtenteils emanzipierend verstandene Vereinigung mit sich selbst im Freitodentschluss akzentuierend, wurden Bücher vorgestellt, die, recht artifiziell die Grenzen der Genres auslotend, den Freitod an die Schwelle zwischen Leben und Kunst, Realität und Fiktion, Autor und Text, Text und Nichttext sowie diverse Textebenen situiert haben. Allerdings bereits im Triptychon spürt man die Machtlosigkeit, auf die auf die man sich gefasst machen muss, sobald man die Perspektive verlässt, in der jedes individuelle Leben über die dem Text zugrundeliegenden Zeitrhythmen entscheidet. Einmal im Kreislauf des ewig Gewesenen verhaftet, das selbst über die Grenzen des individuellen Lebens hinausreicht, und somit den Freitod zwecklos macht, haben die Figuren nichts, woran sie ihre Hoffnung und Erwartung binden könnten.

Oben genannte Prosatexte nähern sich dem Freitodthema eben von dieser Seite her. Suizid wird in ihnen nicht als die Tat eines Einzelnen von Bedeutung; zentral für diese Texte ist, dass sie den Menschen aus seinen individuellen Zeitrhythmen herausnehmen, um ihn in andere, viel längere Zeiträume hineinzuversetzen. In diesen kosmischen, evolutionären, oder geologischen Zeiträumen erscheint folglich das Leben des Menschen, samt dem individuellen Tod, ja selbst samt dem kollektiven Verschwinden der menschlichen Gattung als etwas weniger Gewichtiges, ja Marginales und Periphäres. Die Perspektive des Menschen, sei er noch am Leben, oder schon tot, verliert an Relevanz. Kurz: Hier wird in unterschiedlicher Art der Freitod als das sowieso unausweichliche menschliche Schicksal der verschwindenden oder bereits verschwundenen Spezies imaginiert. Alle drei Texte setzen ihre Maßstäbe nicht vom Menschen her, sondern werden von der transhumanen Perspektive her erzählt. ${ }^{2}$ Bei Ransmayr ist die Rede von einem wissenschaftlichen Projekt, in dem sich der Mensch in einem extra dafür hergestellten Terrarium freiwillig dem Wärmetod aussetzt. Dieser künstlich hergestellte Freitod wird als die ökologisch motivierte Entdeckung des Wesentlichen in dem Moment gedeutet, wo der Mensch nur noch zwischen augenblicklichem oder langsamem Auslöschen wählen kann. Frisch erzählt in seinem Text von einem in den Alpen einsam lebendenden alten Mann, dessen Gedächtnis sichtlich nachlässt, und der, den Tod sehr wohl vor Augen, sich einfach in die großen evolutionären und geologischen Zeiträume hineinversetzt, deren Rhythmen er, nach kurzem Dagegenhalten, nichts mehr entgegenzusetzten bereit

2 Grundlegend zu diesem Thema sind Studien von Georg Braungart und von Wolfgang Riedel. BRAUNGART, Georg (2007): „Katastrophen kennt allein der Mensch, sofern er sie überlebt“. Max Frisch, Peter Handke und die Geologie. In: Carsten Dutt, Roman Luckscheiter ( $\mathrm{Hg}$.): Figurationen der literarischen Moderne. Festschrift für H. Kiesel. Heidelberg, S. 23-41. RIEDEL, Wolfgang (2011): „Der Prozeß der Geschichte ist ein Verbrennen. Erzählte Entropie bei Koeppen und Frisch“. In: Friederike Günther, Torsten Hoffmann (Hg.): Anthropologien der Endlichkeit, Stationen einer literarischen Figur seit der Aufklärung. Göttingen, S. 244-260. 
ist. Er geht in der geologisch ergiebigen Alpenlandschaft um sich herum auf. Sein Tod wird kaum mehr als ein individueller dargestellt, vielmehr wird der Protagonist dadurch zum Bestandteil des natürlichen Prozesses, der ohne den Menschen auskommt. Rumor erzählt eine leicht inzestuöse Vater-Tochter Geschichte, in der vom allmählichen psychischen und physischen Regress des Vaters die Rede ist, der ja auf eine sehr plastische Art wiederum in geologische und biologische Diskurse eingebettet wird. Das transhumane Fazit, das für alle drei gelten könnte, kann in den an Foucault erinnernden Worten dieses Romans wie folgt formuliert werden: „Der Mensch? Ach, Schwamm drüber. Das Menschenkind, die ewige Nummer Eins der Weltgeschichte? Schwamm drüber. Dies Wesen beginnt nun endlich, das Spiel der Regeln zu durchschauen, dem es sein Erscheinen in der Geschichte verdankt. Inzwischen weiß es immerhin so viel, dass dieses selbe Spiel der Regeln es auch wieder aus der Geschichte heraustragen wird. Wenn wir nicht mehr sind, weht noch lange der Wind. Und die Codes gehen ihren unermesslichen Gang. Wir aber versanden, wir werden zugeweht wie ein Scheißhaufen am Strand“ (Strauß 1980, 127).

\section{Literaturliste}

BRAUNGART, Georg (2007): „Katastrophen kennt allein der Mensch, sofern er sie überlebt“. Max Frisch, Peter Handke und die Geologie. In: Dutt, Carsten, Luckscheiter, Roman (Hg.): Figurationen der literarischen Moderne. Festschrift für H. Kiesel. Heidelberg, S. 23-41.

CANETTI, Elias (1994): Die Blendung. Frankfurt/M. Fischer.

DEMETZ, Peter (1988): Fette Jahre, magere Jahre: deutschsprachige Literatur von 1965 bis 1985. München.

HILDESHEIMER, Wolfgang (1981): Marbot. Eine Biographie. Frankfurt/M.: Suhrkamp.

HILDESHEIMER, Wolfgang (1981b): „Kunst- und Wunschfigur inmitten der Geschichte. Wolfgang Hildesheimer im Gespräch über seine Biographie Marbot. (W. F. Schoeller). Frankfurter Rundschau, 15. 10. 1981.

HILDESHEIMER, Wolfgang (1988): „Die Subjektivität des Biographen“. In: ders.: Das Ende der Fiktionen. Reden aus fünfundzwanzig Jahren. Frankfurt/Main, S. 123-138.

KLEIN, Christian (2009): Handbuch Biographie. Methoden, Traditionen, Theorien. Stuttgart Weimar.

MAGENAU, Jörg (2002): Christa Wolf. Eine Biographie. Berlin.

NEUMANN, Peter Horst (1986): „Hildesheimers Ziel und Ende. Über Marbot und Folgerichtigkeit des Gesamtwerkes. In: Text und Kritik (89/90) Wolfgang Hildesheimer. München, S. 20-32.

NIETZSCHE, Friedrich (1954): Fröhliche Wissenschaft. Werke in drei Bänden. Band II. München. RESCHKE, Karin (1982): Verfolgte des Glücks. Findebuch der Henriette Vogel. Hamburg: Rotbuch.

RIEDEL, Wolfgang (2011): „Der Prozeß der Geschichte ist ein Verbrennen. Erzählte Entropie bei Koeppen und Frisch“. In: Friederike Günther, Hoffmann, Torsten (Hg.): Anthropologien der Endlichkeit, Stationen einer literarischen Figur seit der Aufklärung. Göttingen, S. 244-260.

STRAUß, Botho (1975): Marlenes Schwester. Theorie der Drohung. Zwei Erzählungen. Hamburg: Hanser.

STRAUß, Botho (1980): Rumor. Hamburg. 
VESPER, Bernward (1983): Die Reise. Romanessay. Hamburg. WOLF, Christa (1981): Kein Ort. Nirgends. Hamburg: [1979].

doc. Mgr. Aleš Urválek, Ph.D. / urvalek@phil.muni.cz

Masarykova univerzita, Filozofická fakulta, Ústav germanistiky, nordistiky a nederlandistiky Arna Nováka 1, 60200 Brno, CZ 